\title{
The synchronization of instantaneously coupled harmonic oscillators using sampled data with measurement noise
}

\author{
Jingyi Wang ${ }^{a}$ Jianwen Feng ${ }^{\mathrm{b}, 1}$ Chen Xu ${ }^{\text {b,1 }}$ Michael Z. Q. Chen ${ }^{\mathrm{c}}$ Yi Zhao $^{\mathrm{b}}$ \\ Jiqiang Feng ${ }^{b}$ \\ ${ }^{\mathrm{a}}$ Key Laboratory of Optoelectronic Devices and Systems of Ministry of Education and Guangdong Province, and College of \\ Optoelectronic Engineering, Shenzhen University, Shenzhen 518060, PR China \\ ${ }^{\mathrm{b}}$ College of Mathematics and Computational Science, Shenzhen University, Shenzhen 518060, PR China \\ ${ }^{\mathrm{c}}$ Department of Mechanical Engineering, The University of Hong Kong, Hong Kong
}

\begin{abstract}
In this brief, we propose an effective algorithm for synchronization of instantaneously coupled harmonic oscillators by using sampled data which may contain measurement noise. We discuss the convergence of this algorithm for both fixed and switching directed network topologies in the presence or absence of leaders. We also establish sufficient conditions under which the coupled harmonic oscillators could attain synchronized oscillatory motions in the presence of noise. And moreover, it is shown that synchronization can be attained even when the velocity information is exchanged only at discrete-time instants. Finally, we give some numerical examples to illustrate the effectiveness of the theoretical results.
\end{abstract}

Key words: Synchronization, coupled harmonic oscillators, instantaneous interactions, sampled data, measurement noise

\section{Introduction}

Synchronization phenomena are common in nature and hold particular promise for applications to many fields (for example, population dynamics, power systems and automatic control, see Boccaletti et al. (2002); Dörfler and Bullo (2014); Tanner et al. (2007); Wang and Chen (2002); Wang et al. (2015b)). Generally speaking, synchronization is the process in which two or more (coupled) dynamical systems seek to adjust a certain prescribed property of their motions to a common behavior in the limit as time tends to infinity (Boccaletti et al. (2002)). Many studies have therefore been conducted on these problems (see Boccaletti et al. (2002); Dörfler

\footnotetext{
‡ This work was supported by the National Science Foundation of China under Grant No. 61273220, 61373087, $61374053,61377017,61472257$ and 61401283 . This paper has never been presented at any IFAC meetings.

Email addresses: jingyi.jy.wang@gmail.com (Jingyi

Wang), fengjw@szu.edu.cn (Jianwen Feng),

xuchen_szu@szu.edu.cn (Chen Xu), mzqchen@hku.hk

(Michael Z. Q. Chen), yzhao@szu.edu.cn (Yi Zhao),

fengjq@szu.edu.cn (Jiqiang Feng).

1 Tel.: +86 755 26532312; Fax: +86 75526538881 .
}

and Bullo (2014); Olfati-Saber et al. (2007); Wang et al. (2015a) and the references therein) because of their great relevance to real world applications.

Coupled harmonic oscillators are interesting because both their frequencies and amplitudes can be made to converge to the same values over time, irrespective of their initial displacements (Ballard et al. (2010)). As a result, some authors have devoted to investigating different synchronization algorithms for coupled harmonic oscillators from various perspectives. For instance, Ren (2008) studied the synchronization of continuous-time coupled harmonic oscillators with local interactions over both fixed and switching network topologies, while Ballard et al. (2010) also addressed discrete-time coupled harmonic oscillators and applied it to the synchronized motions of multiple mobile robots. In addition, Zhou et al. (2012) considered the synchronization of coupled harmonic oscillators with local instantaneous interactions and obtained some sufficient conditions for fixed and switching topologies in either the presence or the absence of leaders. Zhang and Zhou (2012) also investigated the synchronization of undirected networks of coupled harmonic oscillators using sampled data measurements with controller failures. Zhou et al. (2013) 
further addressed sampled data synchronization of coupled harmonic oscillators with controller failure and communication delays. Furthermore, Sun et al. (2014) investigated the synchronization of instantaneously coupled harmonic oscillators with directed topology. Recently, Sun et al. (2015) focused on synchronization of coupled harmonic oscillators in the presence of random noise. In particular, the synchronization of coupled harmonic oscillators plays an important role in applications such as motion coordination, consensus seeking and collective tracking, see Casau et al. (2015); Chiorescu et al. (2004); Li and Ding (2015); Ren and Beard (2005); Ren (2008); Zhang et al. (2013); Zhou et al. (2012).

With the aid of high-speed computers and modern control systems and signal processing techniques, samples of the control input signals need only be taken at discretetime instants. Meanwhile, many synchronization studies of dynamical networks have been performed recently using sampled data as the main means of information transfer among the network agents. For example, synchronization of coupled harmonic oscillators was considered via sampled data control by Sun et al. (2014); Zhou et al. (2013). In addition, sampled data synchronization control of dynamical networks was studied by stochastic sampling by Shen et al. (2012) and aperiodic sampling by Wen et al. (2014). No studies, however, have ever been conducted on the synchronization of instantaneously coupled harmonic oscillators using sampled data that contains measurement noise.

In this paper, we consider the dynamic behaviors of a set of identical harmonic oscillators. The main aim of this paper is to identify and analyze the synchronization conditions for instantaneously coupled harmonic oscillators using only sampled velocity data with measurement noise in either the presence or the absence of leaders. The communication topology of the proposed algorithm is directed, because the sampling cost of algorithms with directed information flow is smaller than their undirected counterparts. The convergence analysis uses tools as algebraic graph theory, matrix theory, statistics and impulsive differential equations, and the theoretical results are then applied to collective tracking of some specific multi-agent systems.

The rest of this paper is organized as follows. In Section 2, we introduce the general model for a system of instantaneously coupled harmonic oscillators with measurement noise. In Section 3, we establish some synchronization criteria for such networks when leaders are either present or absent and we illustrate the theoretical results with some numerical examples in Section 4. Finally, conclusions are drawn in Section 5.

\section{Preliminaries}

First, we write down the notations that will be used throughout this paper. Let $\mathbf{1}_{n}=[1,1, \ldots, 1]^{\top} \in \mathbb{R}^{n}$ and $I_{n}$ be the $n$-dimensional identity matrix and we use the superscript $T$ to denote the transpose of a matrix and $\rho(\cdot)$ denotes the spectral radius. We write $x \doteq y$ to mean that $x$ is defined to be another name for $y$. Finally, for square matrices $A$ and $B$, we write $A \succ B$ (respectively, $\succeq, \prec$ and $\preceq$ ) to mean that $A-B$ is positive definite (respectively, positive semi-definite, negative definite and negative semi-definite).

To analyze the convergence conditions for a set of coupled harmonic oscillators over any arbitrary network topology, the oscillator interactions shall be modeled by a directed graph. Let $\mathcal{G}=(\mathcal{V}, \mathcal{E})$ be a directed graph for some set of nodes $\mathcal{V}=\{1,2, \ldots, n\}$ and set of edges $\mathcal{E} \subseteq \mathcal{V} \times \mathcal{V}$. And denote $A=\left[a_{i j}\right] \in \mathbb{R}^{n \times n}$ as the adjacency matrix associated with $\mathcal{G}$ such that $a_{i j}$ is a positive weight for all $(i, j) \in \mathcal{E}$ (so that node $i$ receives information from node $j$ while $j$ being called the parent of $i)$ and $a_{i j}=0$ for all $(i, j) \notin \mathcal{E}$. Define the Laplacian matrix $L=\left[l_{i j}\right] \in \mathbb{R}^{n \times n}$ associated with $\mathcal{G}$ by $l_{i i}=\sum_{i=1, j \neq i}^{n} l_{i j}$, where $l_{i j}=-a_{i j}(\leq 0)$ for $i \neq j$. A sequence of edges $\left(i_{1}, i_{2}\right),\left(i_{2}, i_{3}\right), \ldots$, for $i_{j} \in \mathcal{V}$, is called a directed path of $\mathcal{G}$. And a directed graph is strongly connected if there is a directed path from every node to every other node. Moreover, a directed graph is balanced if $\sum_{j=1}^{n} a_{i j}=\sum_{j=1}^{n} a_{j i}$ for every node $i$.

The measured values used in the sampled data control methods (Zhang and Shi (2012)), however, are usually contaminated with measurement noise arising from the measuring errors of the physical devices and the various states of the observers (for instance, the observers' relative positions to the instruments and so on). In Fig. 1, let the points $j\left(x_{j}, y_{j}\right), i\left(x_{i}, y_{i}\right)$ and $\tilde{i}\left(x_{\tilde{i}}, y_{\tilde{i}}\right)$ be the state of the observer, the state of the (expected) observed node and the actual measured state of the observed node, respectively. Let $\overrightarrow{j i}, \overrightarrow{j i}$ and $\vec{i} \vec{i}$ be the real difference, the measured difference and the measurement noise, respectively, and $\omega=\left[\omega_{x}, \omega_{y}\right]^{\top} \in \mathbb{R}^{2}$ be a random noise vector. Clearly, $\overrightarrow{j i}=\overrightarrow{j i}+\vec{i} \vec{i}$. More precisely, we have

$$
\left[\begin{array}{c}
x_{\tilde{i}}-x_{j} \\
y_{\tilde{i}}-y_{j}
\end{array}\right]=\left[\begin{array}{l}
x_{i}-x_{j} \\
y_{i}-y_{j}
\end{array}\right]+\left[\begin{array}{l}
\omega_{x} \\
\omega_{y}
\end{array}\right] \circ\left[\begin{array}{l}
x_{i}-x_{j} \\
y_{i}-y_{j}
\end{array}\right],
$$

where $\circ$ is the Hadamard product.

Motivated by the aforementioned comments and taking sampled data noise into account, we propose a highly effective algorithm for synchronizing $n$ instantaneously coupled harmonic oscillators that are described by the 


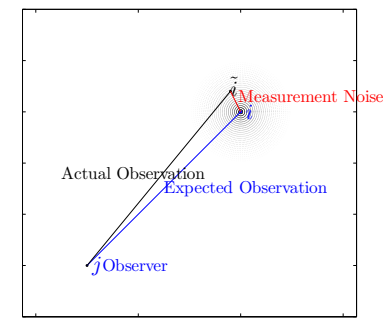

Fig. 1. A 2D sketch of sampled data containing measurement noise.

impulsive differential equations

$$
\begin{cases}\dot{r}_{i}(t)=v_{i}(t), \quad \dot{v}_{i}(t)=-\alpha r_{i}(t), & t \neq t_{k}, \\ \Delta r_{i}\left(t_{k}\right)=0, & t=t_{k}, \\ \Delta v_{i}\left(t_{k}\right)=\sum_{j=1}^{n} a_{i j}\left[\mu\left(v_{j}\left(t_{k}\right)-v_{i}\left(t_{k}\right)\right)\right. & \\ \left.+\omega_{k}\left(v_{j}\left(t_{k}\right)-v_{i}\left(t_{k}\right)\right)\right], & t=t_{k},\end{cases}
$$

$i=1,2, \ldots, n$, for some positive constant $\alpha$, where $r(t) \in$ $\mathbb{R}$ is the position of the oscillator and $v(t) \in \mathbb{R}$ is its velocity, the coupling strength $\mu>0$, the coefficients $a_{i j} \geq 0$ if and only if oscillator $i$ can attain the velocity of oscillator $j$, the time sequence $\left\{t_{k}\right\}_{k=1}^{+\infty}$ satisfying $0=$ $t_{0}<t_{1}<\cdots<t_{k}<\cdots, \lim _{k \rightarrow \infty} t_{k}=+\infty, \Delta r_{i}\left(t_{k}\right)=$ $r_{i}\left(t_{k}^{+}\right)-r_{i}\left(t_{k}\right), \Delta v_{i}\left(t_{k}\right)=v_{i}\left(t_{k}^{+}\right)-v_{i}\left(t_{k}\right)$ with $r_{i}\left(t_{k}^{+}\right)=$ $\lim _{t \rightarrow t_{k}^{+}} r_{i}(t), v_{i}\left(t_{k}^{+}\right)=\lim _{t \rightarrow t_{k}^{+}} v_{i}(t), \lim _{t \rightarrow t_{k}^{-}} r_{i}(t)=$ $r_{i}\left(t_{k}\right)$ and $\lim _{t \rightarrow t_{k}^{-}} v_{i}(t)=v_{i}\left(t_{k}\right)$ (so that $r_{i}(t)$ and $v_{i}(t)$ are left continuous), the sequence of random variables $\left\{\omega_{k}\right\}$ is independent and identically distributed with zero-mean and $\sigma^{2}$ variance and the part $\omega_{k}\left(v_{i}\left(t_{k}\right)-\right.$ $\left.v_{j}\left(t_{k}\right)\right)$ is the measurement noise and depends on the states of oscillators $i$ and $j$ for $k \in \mathbb{N}$.

\section{Synchronization analysis}

\subsection{Synchronization without a leader}

Theorem 1 If the directed graph $\mathcal{G}$ is strongly connected and

(1) $0<\lambda_{n}<\frac{(1-1 / \alpha) \sin ^{2}\left(\sqrt{\alpha} T_{k}\right) \zeta_{n}+1-\zeta_{n}}{(\alpha-1) \sin ^{2}\left(\sqrt{\alpha} T_{k}\right)+1-\zeta_{n}}$;

(2) $\alpha>1+\frac{\zeta_{n}-1}{1-\zeta_{n}+\zeta_{n} \sin ^{2}\left(\sqrt{\alpha} T_{k}\right)}>0$;

(3) $T_{k} \neq \frac{j \pi}{\sqrt{\alpha}}$, for all $k \in \mathbb{N}$ and $j \in \mathbb{N}$,

where $\zeta_{n}>0$ and $\lambda_{n}>0$ are the greatest eigenvalues of $\left(I_{n}-\Xi\right)^{\top}\left(I_{n}-\Xi\right)$ and $\left(I_{n}-\Xi-\mu L\right)^{\top}\left(I_{n}-\Xi-\mu L\right)+$ $\sigma^{2} L^{\top} L$, respectively, then the solutions $\left[r_{i}(t), v_{i}(t)\right]^{\top}$ of system (1) converge in mean square to the synchronized state

$$
\left[\begin{array}{l}
\gamma(t) \\
\nu(t)
\end{array}\right]=\left[\begin{array}{c}
\xi^{\top} r(0) \cos (\sqrt{\alpha} t)+\frac{1}{\sqrt{\alpha}} \xi^{\top} v(0) \sin (\sqrt{\alpha} t) \\
-\sqrt{\alpha} \xi^{\top} r(0) \sin (\sqrt{\alpha} t)+\xi^{\top} v(0) \cos (\sqrt{\alpha} t)
\end{array}\right]
$$

where $\xi=\left[\xi_{1}, \xi_{2}, \ldots, \xi_{n}\right]^{\top}\left(\sum_{i=1}^{n} \xi_{i}=1\right)$ is a left eigenvector of $L$ corresponding to the eigenvalue $0, \Xi=\mathbf{1}_{n} \xi^{\top}$ and $r(0)$ and $v(0)$ are the initial values of system (1).

PROOF. Rewrite equation (1) into the following form

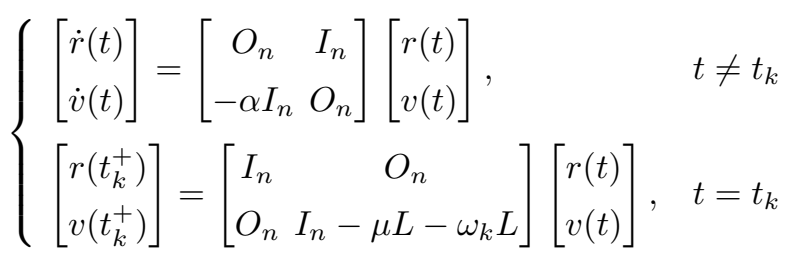

and let $\left[r^{\top}(t), v^{\top}(t)\right]^{\top}$ be the solution of (3) with initial value $\left[r^{\top}(0), v^{\top}(0)\right]^{\top}$. Then write

$$
\left[\begin{array}{c}
\dot{\gamma}(t) \\
\dot{\nu}(t)
\end{array}\right]=\left[\begin{array}{cc}
0 & 1 \\
-\alpha & 0
\end{array}\right]\left[\begin{array}{l}
\gamma(t) \\
\nu(t)
\end{array}\right]
$$

and, noting that $[\gamma(t), \nu(t)]^{\top}$ in $(2)$ is the solution of (4) with initial value $\left[\xi^{\top} r(0), \xi^{\top} v(0)\right]^{\top}$, introduce the synchronization error

$$
\left[\begin{array}{l}
e(t) \\
s(t)
\end{array}\right]=\left[\begin{array}{l}
r(t) \\
v(t)
\end{array}\right]-\left[\begin{array}{l}
\gamma(t) \mathbf{1}_{n} \\
\nu(t) \mathbf{1}_{n}
\end{array}\right]
$$

It is clear, from equations (3) and (4), that $\left[e^{\top}(t), s^{\top}(t)\right]^{\top}$ is a solution of equation (3) corresponding to the initial value

$$
\left[\begin{array}{l}
e(0) \\
s(0)
\end{array}\right]=\left[\begin{array}{cc}
I_{n}-\Xi & O_{n} \\
O_{n} & I_{n}-\Xi
\end{array}\right]\left[\begin{array}{l}
r(0) \\
v(0)
\end{array}\right]
$$

and so it follows from the theory of impulsive differential equations (Yang (2001)) that

$$
\begin{aligned}
& {\left[\begin{array}{l}
e(t) \\
s(t)
\end{array}\right]=\exp \left(\left[\begin{array}{cc}
O_{n} & I_{n} \\
-\alpha I_{n} & O_{n}
\end{array}\right]\left(t-t_{k-1}\right)\right)} \\
& \times \prod_{j=1}^{k-1}\left\{\left[\begin{array}{cc}
I_{n} & O_{n} \\
O_{n} & I_{n}-\left(\mu+\omega_{k-j}\right) L
\end{array}\right] \exp \left(\left[\begin{array}{cc}
O_{n} & I_{n} \\
-\alpha I_{n} & O_{n}
\end{array}\right] T_{k-j}\right)\right\} \\
& \times\left[\begin{array}{cc}
I_{n}-\Xi & O_{n} \\
O_{n} & I_{n}-\Xi
\end{array}\right]\left[\begin{array}{l}
r(0) \\
v(0)
\end{array}\right], \quad t \in\left(t_{k-1}, t_{k}\right], \quad k \geq 2,
\end{aligned}
$$


$\exp \left(\left[\begin{array}{cc}O_{n} & I_{n} \\ -\alpha I_{n} & O_{n}\end{array}\right] s\right)=\left[\begin{array}{cc}\cos (\sqrt{\alpha} s) I_{n} & \frac{1}{\sqrt{\alpha}} \sin (\sqrt{\alpha} s) I_{n} \\ -\sqrt{\alpha} \sin (\sqrt{\alpha} s) I_{n} & \cos (\sqrt{\alpha} s) I_{n}\end{array}\right] . \quad \mathbb{E}\left\{\left[\begin{array}{c}e\left(t_{1}^{+}\right) \\ s\left(t_{1}^{+}\right)\end{array}\right]\right\}=\mathbb{E}\left[B_{1}\right] \exp \left(A T_{1}\right)\left[\begin{array}{c}r(0) \\ v(0)\end{array}\right]$

and the covariance matrix

Now we know, by definition of $\Xi$ and $L$, that $\Xi L=L \Xi=$ $O_{n}, \Xi^{2}=\Xi\left(I_{n}-\Xi\right)^{2}=I_{n}-\Xi,\left(I_{n}-L\right)\left(I_{n}-\Xi\right)=$ $I_{n}-L-\Xi$ and $\left(I_{n}-\Xi\right)\left(I_{n}-L-\Xi\right)=I_{n}-L-\Xi$ and that $\left[e^{\top}(t), s^{\top}(t)\right]^{\top}$ is just a solution of the impulsive differential equations (with noise)

$$
\begin{aligned}
& \operatorname{cov}\left\{\left[\begin{array}{l}
e\left(t_{1}^{+}\right) \\
s\left(t_{1}^{+}\right)
\end{array}\right]\right\}=\mathbb{E}\left\{\omega_{1} \tilde{L} \exp \left(A T_{1}\right)\left[\begin{array}{l}
r(0) \\
v(0)
\end{array}\right]\right. \\
& \left.\times\left[\begin{array}{l}
r(0) \\
v(0)
\end{array}\right]^{\top} \exp \left(A^{\top} T_{1}\right) \omega_{1} \tilde{L}^{\top}\right\},
\end{aligned}
$$

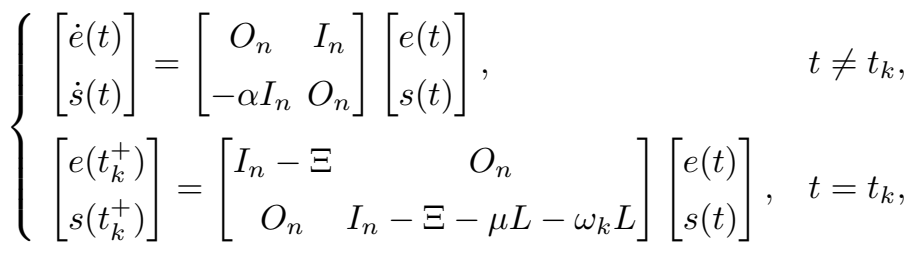

and by mathematical calculations (see Section 6.2 of Petersen and Pedersen (2012)), one can get

with initial condition $\left[e(0)^{\top}, s(0)^{\top}\right]=\left[r(0)^{\top}, v(0)^{\top}\right]$ (see Zhou et al. (2012)). Therefore, by letting

$$
\begin{aligned}
A & =\left[\begin{array}{cc}
O_{n} & I_{n} \\
-\alpha I_{n} & O_{n}
\end{array}\right], \quad \tilde{L}=\left[\begin{array}{cc}
O_{n} & O_{n} \\
O_{n} & L
\end{array}\right], \\
B & =\left[\begin{array}{cc}
I_{n}-\Xi & O_{n} \\
O_{n} & I_{n}-\Xi-\mu L
\end{array}\right]
\end{aligned}
$$

and $B_{k}=B-\omega_{k} \tilde{L}$, one can get $\mathbb{E}\left[B_{k}\right]=B$ and $\mathbb{E}\left[B_{k}^{\top} B_{k}\right]=\operatorname{diag}\left\{B_{11}, B_{22}\right\}$, where $B_{11}=\left(I_{n}-\right.$ $\Xi)^{\top}\left(I_{n}-\Xi\right)$ and $B_{22}=\left(I_{n}-\Xi-\mu L\right)^{\top}\left(I_{n}-\Xi-\mu L\right)+$ $\sigma^{2} L^{\dagger} L$. So that, for all $t \in\left[t_{k-1}, t_{k}\right)$, we have

$$
\left\{\begin{array}{l}
e_{i}(t)=\cos (\sqrt{\alpha} t) e_{i}\left(t_{k-1}^{+}\right)+\frac{1}{\sqrt{\alpha}} \sin (\sqrt{\alpha} t) s_{i}\left(t_{k-1}^{+}\right) \\
\left.s_{i}(t)=-\sqrt{\alpha} \sin (\sqrt{\alpha} t) e_{i}\left(t_{k-1}^{+}\right)+\cos (\sqrt{\alpha} t) s_{i}\left(t_{k-1}^{+}\right)\right) .
\end{array}\right.
$$

Furthermore, it is clear that

$e_{i}(t)^{\top} e_{i}(t)+\frac{1}{\alpha} s_{i}(t)^{\top} s_{i}(t)=\Delta_{i, k}$, for $t \in\left[t_{k-1}, t_{k}\right)$,

where $\Delta_{i, k}=e_{i}\left(t_{k-1}^{+}\right)^{\top} e_{i}\left(t_{k-1}^{+}\right)+\frac{1}{\alpha} s_{i}\left(t_{k-1}^{+}\right)^{\top} s_{i}\left(t_{k-1}^{+}\right)$. It now suffices to show that $\sum_{i=1}^{n} \Delta_{i, k} \rightarrow 0$ as $k \rightarrow+\infty$ achieves synchronization.

When $t=t_{1}^{+}$, we have

$$
\left[\begin{array}{l}
e\left(t_{1}^{+}\right) \\
s\left(t_{1}^{+}\right)
\end{array}\right]=B_{1} \exp \left(A T_{1}\right)\left[\begin{array}{l}
r(0) \\
v(0)
\end{array}\right]
$$

$$
\begin{aligned}
& \mathbb{E}\left\{\left[\begin{array}{l}
e\left(t_{1}^{+}\right) \\
s\left(t_{1}^{+}\right)
\end{array}\right]^{\top}\left[\begin{array}{l}
e\left(t_{1}^{+}\right) \\
s\left(t_{1}^{+}\right)
\end{array}\right]\right\} \\
\leq & \rho\left(\mathbb{E}\left\{e^{A^{\top} T_{1}} B_{1}^{\top} B_{1} e^{A T_{1}}\right\}\right)\left[\begin{array}{l}
r(0) \\
v(0)
\end{array}\right]^{\top}\left[\begin{array}{l}
r(0) \\
v(0)
\end{array}\right] .
\end{aligned}
$$

When $t=t_{2}^{+}$, in the similar way, we obtain

$$
\begin{aligned}
& \mathbb{E}\left\{\left[\begin{array}{l}
e\left(t_{2}^{+}\right) \\
s\left(t_{2}^{+}\right)
\end{array}\right]^{\top}\left[\begin{array}{l}
e\left(t_{2}^{+}\right) \\
s\left(t_{2}^{+}\right)
\end{array}\right]\right\} \\
\leq & \rho\left(\mathbb{E}\left\{e^{A^{\top} T_{2}} B_{2}^{\top} B_{2} e^{A T_{2}}\right\}\right) \mathbb{E}\left\{\left[\begin{array}{l}
e\left(t_{1}^{+}\right) \\
s\left(t_{1}^{+}\right)
\end{array}\right]^{\top}\left[\begin{array}{l}
e\left(t_{1}^{+}\right) \\
s\left(t_{1}^{+}\right)
\end{array}\right]\right\} .
\end{aligned}
$$

It therefore follows by induction that

$$
\begin{aligned}
& \mathbb{E}\left\{\left[\begin{array}{l}
e\left(t_{k}^{+}\right) \\
s\left(t_{k}^{+}\right)
\end{array}\right]^{\top}\left[\begin{array}{l}
e\left(t_{k}^{+}\right) \\
s\left(t_{k}^{+}\right)
\end{array}\right]\right\} \\
& \leq \rho\left(\mathbb{E}\left\{e^{A^{\top} T_{k}} B_{k}^{\top} B_{k} e^{A T_{k}}\right\}\right) \mathbb{E}\left\{\left[\begin{array}{l}
e\left(t_{k-1}^{+}\right) \\
s\left(t_{k-1}^{+}\right)
\end{array}\right]^{\top}\left[\begin{array}{c}
e\left(t_{k-1}^{+}\right) \\
s\left(t_{k-1}^{+}\right)
\end{array}\right]\right\}
\end{aligned}
$$

and we must now show that $\rho\left(\mathbb{E}\left\{e^{A^{\top} T_{k}} B_{k}^{\top} B_{k} e^{A T_{k}}\right\}\right)<$ 1.

There exist unitary matrices $U$ and $V$ such that $U^{\top}\left(I_{n}-\Xi\right)^{\top}\left(I_{n}-\Xi\right) U=\operatorname{diag}\left\{0, \zeta_{2}, \ldots, \zeta_{n}\right\}=H$ and $V^{\top}\left(\left(I_{n}-\Xi-\mu L\right)^{\top}\left(I_{n}-\Xi-\mu L\right)+\sigma^{2} L^{\top} L\right) V=$ $\operatorname{diag}\left\{0, \lambda_{2}, \ldots, \lambda_{n}\right\}=\Lambda, \zeta_{i} \geq \zeta_{i-1}>0, \lambda_{i} \geq \lambda_{i-1}>0$ for $i=3, \ldots, n$. By letting $\tilde{\Lambda}=\operatorname{diag}\left\{0, \lambda_{n}, \ldots, \lambda_{n}\right\} \in$ $\mathbb{R}^{n \times n}$ and $\tilde{H}=\operatorname{diag}\left\{0, \zeta_{n}, \ldots, \zeta_{n}\right\}$, we have

$$
\operatorname{diag}\left\{U^{\top}, V^{\top}\right\} \mathbb{E}\left\{B_{k}^{\top} B_{k}\right\} \operatorname{diag}\{U, V\}
$$




$$
=\left[\begin{array}{cc}
H & O_{n} \\
O_{n} & \Lambda
\end{array}\right] \preceq\left[\begin{array}{cc}
\tilde{H} & O_{n} \\
O_{n} & \tilde{\Lambda}
\end{array}\right] \doteq \tilde{B} .
$$

Now, it is clear that $\mathbb{E}\left\{e^{A^{\top} T_{k}} B_{k}^{\top} B_{k} e^{A T_{k}}\right\} \preceq e^{A^{\top} T_{k}} \tilde{B} e^{A T_{k}}$ and $\rho\left(\mathbb{E}\left\{e^{A^{\top} T_{k}} B_{k}^{\top} B_{k} e^{A T_{k}}\right\}\right) \leq \rho\left(e^{A^{\top} T_{k}} \tilde{B} e^{A T_{k}}\right)$ and so the characteristic equation of $e^{A^{\top} T_{k}} \tilde{B} e^{A T_{k}}$ is

$$
\begin{aligned}
& \operatorname{det}\left(e^{A^{\top} T_{k}} \tilde{B} e^{A T_{k}}-x I_{2 n}\right) \\
= & x^{2} \prod_{i=2}^{n}\left\{x^{2}-\left(\frac{1}{\alpha} \zeta_{n} \sin ^{2}\left(\sqrt{\alpha} T_{k}\right)+\zeta_{n} \cos ^{2}\left(\sqrt{\alpha} T_{k}\right)\right.\right. \\
& \left.\left.+\lambda_{n} \cos ^{2}\left(\sqrt{\alpha} T_{k}\right)+\alpha \lambda_{n} \sin ^{2}\left(\sqrt{\alpha} T_{k}\right)\right) x+\lambda_{n} \zeta_{n}\right\}
\end{aligned}
$$

with roots (i.e. eigenvalues) $\lambda_{(1) \pm}\left(\mathbb{E}\left\{e^{A^{\top} T_{k}} B_{k}^{\top} B_{k} e^{A T_{k}}\right\}\right)=$ 0 and $\lambda_{(i) \pm}\left(\mathbb{E}\left\{e^{A^{\top} T_{k}} B_{k}^{\top} B_{k} e^{A T_{k}}\right\}\right)=\frac{1}{2}\left(b \pm \sqrt{b^{2}-4 \lambda_{n} \zeta_{n}}\right)$, $i=2,3, \ldots, n$, where $b=\frac{1}{\alpha} \zeta_{n} \sin ^{2}\left(\sqrt{\alpha} T_{k}\right)+\zeta_{n} \cos ^{2}\left(\sqrt{\alpha} T_{k}\right)+$ $\lambda_{n} \cos ^{2}\left(\sqrt{\alpha} T_{k}\right)+\alpha \lambda_{n} \sin ^{2}\left(\sqrt{\alpha} T_{k}\right)$. Since

$\left|\lambda_{(i) \pm}\left(\mathbb{E}\left\{e^{A^{\top} T_{k}} B_{k}^{\top} B_{k} e^{A T_{k}}\right\}\right)\right|=\frac{1}{2}\left(b \pm \sqrt{b^{2}-4 \lambda_{n} \zeta_{n}}\right)$,

when $b^{2} \geq 4 \lambda_{n} \zeta_{n}$ and

$$
\left|\lambda_{(i) \pm}\left(\mathbb{E}\left\{e^{A^{\top} T_{k}} B_{k}^{\top} B_{k} e^{A T_{k}}\right\}\right)\right|=\sqrt{\lambda_{n} \zeta_{n}},
$$

when $b^{2}<4 \lambda_{n} \zeta_{n}$, we have $\left|\lambda_{(i) \pm}\left(\mathbb{E}\left\{e^{A^{\top} T_{k}} B_{k}^{\top} B_{k} e^{A T_{k}}\right\}\right)\right|<$ 1 when $b<1+\lambda_{n} \zeta_{n}$ and $\lambda_{n} \zeta_{n}<1$.

Also, it is clear that $\left(\left(\frac{1}{\alpha}-1\right) \sin ^{2}\left(\sqrt{\alpha} T_{k}\right) \zeta_{n}+\zeta_{n}-1\right) \zeta_{n}<$ $\left((\alpha-1) \sin ^{2}\left(\sqrt{\alpha} T_{k}\right)-\zeta_{n}+1\right)$ and $1+\frac{\zeta_{n}-1}{\sin ^{2}\left(\sqrt{\alpha} T_{k}\right)} \leq$ $1+\frac{\zeta_{n}-1}{1-\zeta_{n}+\zeta_{n} \sin ^{2}\left(\sqrt{\alpha} T_{k}\right)}$ for any $\alpha \in \mathbb{R}, \zeta_{n} \geq 1$ and $\sqrt{\alpha} T_{k} \neq j \pi, j \in \mathbb{N}$ with the equality holding only when $\zeta_{n}=1$. Therefore, since $b<1+\lambda_{n} \zeta_{n}$ and $\lambda_{n} \zeta_{n}<1$ only when conditions (1), (2) and (3) hold, we see that $\rho\left(\mathbb{E}\left\{e^{A^{\top} T_{k}} B_{k}^{\top} B_{k} e^{A T_{k}}\right\}\right) \leq \gamma<1$ and we have

$\lim _{k \rightarrow \infty} \mathbb{E}\left\{\left[\begin{array}{c}e\left(t_{k}^{+}\right) \\ s\left(t_{k}^{+}\right)\end{array}\right]^{\top}\left[\begin{array}{c}e\left(t_{k}^{+}\right) \\ s\left(t_{k}^{+}\right)\end{array}\right]\right\} \leq \lim _{k \rightarrow \infty} \gamma^{k}\left[\begin{array}{l}r(0) \\ v(0)\end{array}\right]^{\top}\left[\begin{array}{l}r(0) \\ v(0)\end{array}\right]=0$.

It now follows from (6) that

$$
\lim _{t \rightarrow \infty} \mathbb{E}\left\{\left[\begin{array}{l}
e(t) \\
s(t)
\end{array}\right]^{\top}\left[\begin{array}{l}
e(t) \\
s(t)
\end{array}\right]\right\}=0
$$

and so all the states $\left(r_{i}(t), v_{i}(t)\right)^{\top}, i=1,2, \ldots, n$ of $(1)$ will asymptotically converge in mean square to the synchronized state $(\gamma(t), \nu(t))^{\top}$. This completes the proof of Theorem 1.
Remark 2 In this study, the communication topology $\mathcal{G}$ does not need to be undirected and its Laplace matrix $L$ is asymmetric. One cannot directly analyze and compare converges by using its eigenvalues, since they may be complex scale. We deal with the problem by using the singular value decomposition, which is less often used to study synchronization in the literature, but is nevertheless extremely useful. Sufficient conditions for synchronization of coupled harmonic oscillators are given in Theorem 1. Because measurement noise directly affects synchronization, it is crucial to find the relationship between the coupling and noise strengths. We can readily compute the values of $\mu$ and $\sigma$ for any given graph $\mathcal{G}$ with the associated Laplacian matrix L. We will also give an example of the relationship between $\mu$ and $\sigma^{2}$ in Fig. 3.

Remark 3 Compared with the existing work concerning synchronization schemes for coupled harmonic oscillators (such as, sampled data control without noise by Sun , et al. (2014); Zhou et al. (2012), and continuous-time coupling without noise by Ren (2008) or with noise in Sun et al. (2015)), a great advantage of the proposed algorithm (1) is that it can deal with the problem based only on the neighbors' relative velocity information in the presence of measurement noise at discrete-time instants, which does not need exact and continuous-time position and velocity information.

We also consider the convergence of coupled harmonic oscillators over switching directed communication topologies without leaders. Let $S_{\mathcal{G}}=\left\{\mathcal{G}_{1}, \ldots, \mathcal{G}_{m}\right\}$ be the set of all possible interconnection graphs at the coupling instant $t=t_{k}$ for $k \in \mathbb{N}$ and let $G_{s(k)} \in S_{\mathcal{G}}$ be the communication topology at $t=t_{k}$ with the corresponding Laplacian $L_{s(k)}$ and adjacent matrix $A_{s(k)}=\left[a_{i j}^{s(k)}\right] \in \mathbb{R}^{n \times n}$, where the map $s(k): N \rightarrow \mathcal{I}=\{1,2, \ldots, m\}$ is a stochastic switching signal that determines the network topology $\mathcal{G}_{s(k)}$ at $t=t_{k}$ for $k \in \mathbb{N}$. In the case we consider the following algorithm

$$
\begin{cases}\dot{r}_{i}(t)=v_{i}(t), \quad \dot{v}_{i}(t)=-\alpha r_{i}(t), & t \neq t_{k}, \\ \Delta r_{i}\left(t_{k}\right)=0, & t=t_{k}, \\ \Delta v_{i}\left(t_{k}\right)=\sum_{j=1}^{n} a_{i j}^{s(k)}\left(\mu+\omega_{k}\right)\left(v_{j}\left(t_{k}\right)-v_{i}\left(t_{k}\right)\right), & t=t_{k} .\end{cases}
$$

where $i=1,2, \ldots, n$. Then, we have the following theorem for the algorithm (9).

Theorem 4 If the directed graphs $\mathcal{G}_{1}, \ldots, \mathcal{G}_{m}$ are balanced and strongly connected and

$$
0<\lambda_{n, \mathcal{I}}<\frac{1}{\alpha}, \quad \alpha>1 \text { and } T_{k} \neq \frac{j \pi}{\sqrt{\alpha}}
$$

for all $k \in \mathbb{N}$ and $j \in \mathbb{N}$, where $\lambda_{n, \mathcal{I}}>0$ are the greatest 
eigenvalues of $\left(I_{n}-\Xi-\mu L_{s}\right)^{\top}\left(I_{n}-\Xi-\mu L_{s}\right)+\sigma^{2} L_{s}^{\top} L_{s}$ for all $s=1,2, \ldots, m$, then the solutions $\left[r_{i}(t), v_{i}(t)\right]^{\top}$ of system (9) will converge in mean square to the synchronized state $[\gamma(t), \nu(t)]^{\top}$ in equation (2), where $\xi=$ $[1 / n, 1 / n, \ldots, 1 / n]^{\top}$ and $r(0)$ and $v(0)$ are the initial values of system (9).

Remark 5 If the directed graphs $\mathcal{G}_{s}$ with the corresponding Laplacian $L_{s}$ are balanced and strongly connected, then $\xi=[1 / n, 1 / n, \ldots, 1 / n]^{\top}$ is a left eigenvector of $L_{s}$ corresponding to the eigenvalue 0 for all $s \in \mathcal{I}$. And $\left(I_{n}-\Xi\right)^{\top}\left(I_{n}-\Xi\right)$ is diagonalizable with a diagonal form $\operatorname{diag}\{0,1,1, \ldots, 1\}$. Based on above, Theorem 4 follows directly from Theorem 1 and the proof is thus omitted for brevity.

\subsection{Synchronization with a leader}

In this subsection, we investigate the situation when an active leader exists with position $\hat{\gamma}(t)$ and velocity $\hat{\mu}(t)$ given by

$$
\dot{\hat{\gamma}}(t)=\hat{\nu}(t), \quad \dot{\hat{\nu}}(t)=-\alpha \hat{\gamma}(t)
$$

In this case, we propose the algorithm

$$
\begin{cases}\dot{r}_{i}(t)=v_{i}(t), \quad \dot{v}_{i}(t)=-\alpha r_{i}(t), & t \neq t_{k}, \\ \Delta r_{i}\left(t_{k}\right)=0, & t=t_{k}, \\ \Delta v_{i}\left(t_{k}\right)=\left(\mu+\omega_{k}\right)\left(\sum _ { j = 1 } ^ { n } a _ { i j } \left(v_{j}\left(t_{k}\right)\right.\right. & \\ \left.\quad-v_{i}\left(t_{k}\right)-d_{i}\left(v_{i}\left(t_{k}\right)-\hat{\nu}\left(t_{k}\right)\right)\right), & t=t_{k},\end{cases}
$$

for $i=1,2, \ldots, n$, where $d_{i}>0$ if $\hat{\nu}\left(t_{k}\right)$ is available to oscillator $i$ at $t=t_{k}(k \in \mathbb{N})$ and $d_{i}=0$ otherwise.

Theorem 6 If the directed graph $\mathcal{G}$ is strongly connected and

$$
0<\hat{\lambda}_{n}<\frac{1}{\alpha}, \alpha>1 \text { and } T_{k} \neq \frac{j \pi}{\sqrt{\alpha}}
$$

for all $k \in \mathbb{N}$ and $j \in \mathbb{N}$, where $\hat{\lambda}_{n}>0$ is the greatest eigenvalue of $\left(I_{n}-\mu(L-D)\right)^{\top}\left(I_{n}-\mu(L-D)\right)+\sigma^{2}(L-$ $D)^{\top}(L-D)$, then the solutions $\left[r_{i}(t), v_{i}(t)\right]^{\top}$ of system (11) will converge in mean square to the synchronized state $[\hat{\gamma}(t), \hat{\nu}(t)]^{\top}$ for any initial values.

PROOF. Consider the synchronization error

$$
\left[\begin{array}{l}
e(t) \\
s(t)
\end{array}\right]=\left[\begin{array}{l}
r(t) \\
v(t)
\end{array}\right]-\left[\begin{array}{l}
\hat{\gamma}(t) \mathbf{1}_{n} \\
\hat{\nu}(t) \mathbf{1}_{n}
\end{array}\right]
$$

one can get

$$
\left[\begin{array}{l}
e(t) \\
s(t)
\end{array}\right]=\mathrm{e}^{A\left(t-t_{k-1}\right)} \otimes I_{n}\left\{\prod_{j=1}^{k-1} \hat{B}_{k} e^{A T_{k-j}}\right\}\left[\begin{array}{l}
e(0) \\
s(0)
\end{array}\right],
$$

for $t \in\left(t_{k-1}, t_{k}\right], k \geq 2$, where

$$
\hat{B}_{k}=\left[\begin{array}{cc}
I_{n} & O_{n} \\
O_{n} & I_{n}-\left(\mu+\omega_{k}\right)(L-D)
\end{array}\right],
$$

$\left[e(0)^{\top}, s(0)^{\top}\right]=\left[\left(r(0)-\hat{\gamma}(0) \mathbf{1}_{n}\right)^{\top},\left(v(0)-\hat{\nu}(0) \mathbf{1}_{n}\right)^{\top}\right]$, $D=\operatorname{diag}\left\{d_{1}, d_{2}, \ldots, d_{n}\right\}$ and one can get

$$
\begin{aligned}
& \mathbb{E}\left\{\left[\begin{array}{l}
e\left(t_{k}^{+}\right) \\
s\left(t_{k}^{+}\right)
\end{array}\right]^{\top}\left[\begin{array}{l}
e\left(t_{k}^{+}\right) \\
s\left(t_{k}^{+}\right)
\end{array}\right]\right\} \\
& \leq \rho\left(\mathbb{E}\left\{e^{A^{\top} T_{k}} \hat{B}_{k}^{\top} \hat{B}_{k} e^{A T_{k}}\right\}\right) \mathbb{E}\left\{\left[\begin{array}{l}
e\left(t_{k-1}^{+}\right) \\
s\left(t_{k-1}^{+}\right)
\end{array}\right]^{\top}\left[\begin{array}{l}
e\left(t_{k-1}^{+}\right) \\
s\left(t_{k-1}^{+}\right)
\end{array}\right]\right\} .
\end{aligned}
$$

Similarly, we have

$$
I_{2} \otimes \hat{V}^{\top} \mathbb{E}\left\{\hat{B}_{k}^{\top} \hat{B}_{k}\right\} I_{2} \otimes \hat{V}=\left[\begin{array}{cc}
I_{n} & O_{n} \\
O_{n} & \hat{\Lambda}
\end{array}\right] \preceq\left[\begin{array}{cc}
I_{n} & O_{n} \\
O_{n} & \hat{\lambda}_{n} I_{n}
\end{array}\right]
$$

where $\hat{\Lambda}$ is a diagonal matrix of the eigenvalues of $\left(I_{n}-\right.$ $\mu(L-D))^{\top}\left(I_{n}-\mu(L-D)\right)+\sigma^{2}(L-D)^{\top}(L-D)$ with $\hat{\lambda}_{n}$ being the greatest one. If condition (12) of Theorem 6 holds, then we have $\rho\left(\mathbb{E}\left\{e^{A^{\top} T_{k}} \hat{B}_{k}^{\top} \hat{B}_{k} e^{A T_{k}}\right\}\right)<1$ and the rest of the proof is similar to that of Theorem 1 . The proof is complete.

Consider the situation where there exists at least one oscillator that is connected to the active leader at $t=t_{k}$ $(k \in \mathbb{N})$ and that different oscillators may be connected to the leader at different instants. In this case, we propose the algorithm

$$
\begin{cases}\dot{r}_{i}(t)=v_{i}(t), \quad \dot{v}_{i}(t)=-\alpha r_{i}(t), & t \neq t_{k}, \\ \Delta r_{i}\left(t_{k}\right)=0, & t=t_{k}, \\ \Delta v_{i}\left(t_{k}\right)=\left(\mu+\omega_{k}\right)\left(\sum _ { j = 1 } ^ { n } a _ { i j } ^ { s ( k ) } \left(v_{j}\left(t_{k}\right)\right.\right. & \\ \left.\left.-v_{i}\left(t_{k}\right)\right)-d_{i}^{s(k)}\left(v_{i}\left(t_{k}\right)-\hat{\nu}\left(t_{k}\right)\right)\right), & t=t_{k},\end{cases}
$$

for $i=1,2, \ldots, n$.

Theorem 7 If the directed graphs $\mathcal{G}_{1}, \ldots, \mathcal{G}_{m}$ are balanced and strongly connected and

$$
0<\hat{\lambda}_{n, \mathcal{I}}<\frac{1}{\alpha}, \quad \alpha>1 \text { and } T_{k} \neq \frac{j \pi}{\sqrt{\alpha}}
$$


for all $k \in \mathbb{N}$ and $j \in \mathbb{N}$, where $\hat{\lambda}_{n, \mathcal{I}}>0$ is the greatest eigenvalue of $\left(I_{n}-\mu\left(L_{s}-D_{s}\right)\right)^{\top}\left(I_{n}-\mu\left(L_{s}-D_{s}\right)\right)+$ $\sigma^{2}\left(L_{s}-D_{s}\right)^{\top}\left(L_{s}-D_{s}\right)$ for all $s \in \mathcal{I}$, then the solutions $\left[r_{i}(t), v_{i}(t)\right]^{\top}$ of system (13) will converge in mean square to the synchronized state $[\hat{\gamma}(t), \hat{\nu}(t)]^{\top}$ for any initial values.

Remark 8 Theorem 7 follows directly from Theorems 4 and 6 , and the proof is thus omitted for brevity.

\section{Numerical Simulations}

In this section, we present some numerical simulations that validate the effectiveness of the theorems in the previous section.

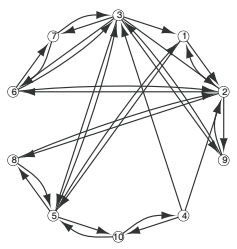

(a) $\mathcal{G}$

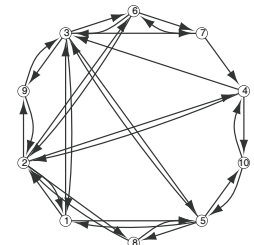

(b) $\mathcal{G}_{1}$

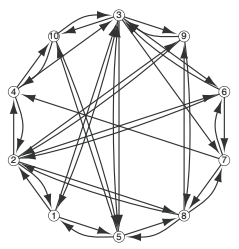

(c) $\mathcal{G}_{2}$
Fig. 2. Directed graph $\mathcal{G}$ and directed and balanced graphs $\mathcal{G}_{1}$ and $\mathcal{G}_{2}$. We assume that $a_{i j}=1$ for all $(j, i) \in \mathcal{E}$ and $a_{i j}=0$ otherwise.

\subsection{Synchronization of coupled harmonic oscillators}

Here we consider a directed network of 10 agents moving in one-dimensional Euclidean space with the topology $\mathcal{G}$ as shown in Fig. 2(a) under algorithm (1). Clearly, graph $\mathcal{G}$ is strongly connected and we choose $\alpha=1.2, T_{k}=1.3$ and $\mu=0.2$ (the set of all the accepted combinations of $\mu$ and $\sigma^{2}$ will be given in Fig. 3). Direct computation yields $\rho\left(e^{A^{\top} T_{k}} B_{k}^{\top} B_{k} e^{A T_{k}}\right)=0.9711$ if $\sigma^{2}=0.003$ so that the conditions of Theorem 1 are satisfied. The evolution of the oscillator states are shown in Fig. 4(a) and Fig. $4(\mathrm{~b})$ for the initial values $r_{i}(0)=8 i-36$, $v_{i}(0)=8 i-32, i=1,2, \ldots, 10$ and the synchronization can be achieved. While one can observe that the synchronization can not be reached with the same parameters except for $\sigma^{2}=0.05$ from Fig. 4(c) and Fig. 4(d). One can see that the measurement noise affects synchronization directly, which cannot be reached when the noise standard deviation is relatively large from this example.

Next, the interaction topology is randomly switched between $\mathcal{G}_{1}$ and $\mathcal{G}_{2}$ (shown as Figs. 2(b) and (2(c))) at time $t_{k}$ and we choose $\alpha=1.2, T_{k}=1.3, \sigma^{2}=0.003$ and $\mu=0.2$. We have $\rho\left(e^{A^{\top} T_{k}} B_{1}^{\top} B_{1} e^{A T_{k}}\right)=0.8393$ and $\rho\left(e^{A^{\top} T_{k}} B_{2}^{\top} B_{2} e^{A T_{k}}\right)=0.8385$. Fig. 5 shows the evolution of the oscillator states under algorithm (9) for the initial values $r_{i}(0)=8 i-36$ and $v_{i}(0)=8 i-32$, $i=1,2, \ldots, 10$.

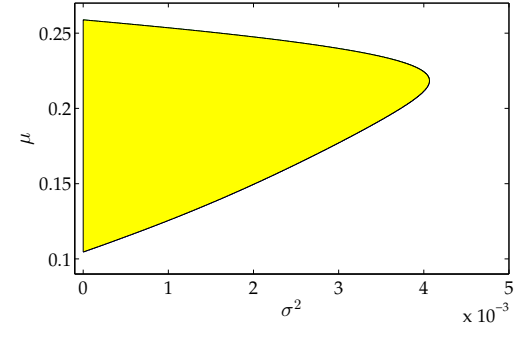

Fig. 3. The relation between coupling and noise strength for $\mathcal{G}$ when $\alpha=1.2$ and $T_{k}=1.3$. The yellow region contains all the accepted combinations such that $\rho\left(e^{A^{\top} T_{k}} B_{k}^{\top} B_{k} e^{A T_{k}}\right)<1$.

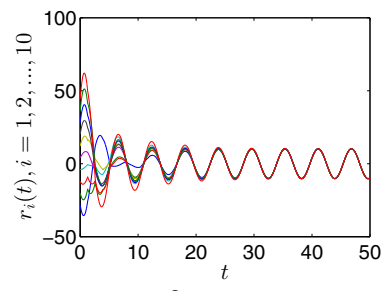

(a) $\sigma^{2}=0.003$

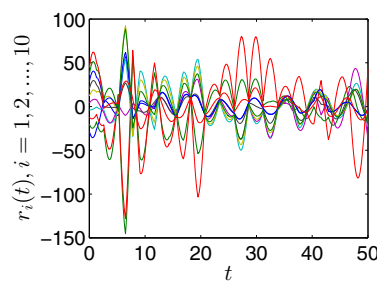

(c) $\sigma^{2}=0.05$

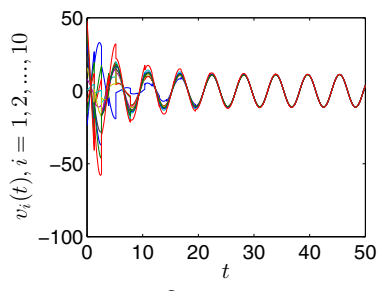

(b) $\sigma^{2}=0.003$

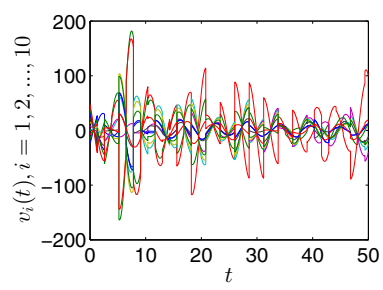

(d) $\sigma^{2}=0.05$
Fig. 4. The time evolution of $r_{i}$ and $v_{i}$ with a fixed interconnection topology for $\mathcal{G}$ and same initial values when $\alpha=1.2$, $T_{k}=1.3 \mu=0.2$, and $\sigma^{2}=0.003(\mathrm{a}, \mathrm{b})$ or $\sigma^{2}=0.05(\mathrm{c}, \mathrm{d})$.

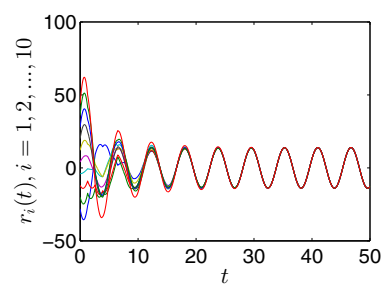

(a)

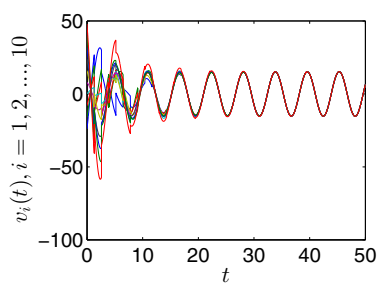

(b)
Fig. 5. The time evolution of $r_{i}$ and $v_{i}$ for switching interconnection topologies $\mathcal{G}_{1}$ and $\mathcal{G}_{2}$ when $\alpha=1.2, T_{k}=1.3$, $\sigma^{2}=0.003$ and $\mu=0.2$.

\subsection{Application to collective tracking}

In the subsection, we apply algorithm (11) to collective tracking a moving object, as implemented by Casau et al. (2015); Li and Ding (2015); Zhang et al. (2013, 2015).

Consider a group of agents with the communication topology as shown in Fig. 2(a). The dynam- 
ics of the object is described by (10) with initial value $\gamma(0)=10$ and $\nu(0)=-10$. Only a subset of agents $(7,8,9,10)$ can access the velocity information of the object. Choose $\alpha=1.2, \sigma^{2}=0.003$, $\mu=0.2, T_{k}=1.3, d_{1}=\cdots=d_{6}=0$ and $d_{7}=\cdots=d_{10}=4.6$. The conditions of Theorem 6 are satisfied and $\rho\left(\mathbb{E}\left(e^{A^{\top} T_{k}} \hat{B}_{k}^{\top} \hat{B}_{k} e^{A T_{k}}\right)\right)=0.9982$. Fig. 6 demonstrates the tracking errors evolving with time. It is obvious that the collective tracking can be achieved, which shows the effectiveness of algorithm (11).

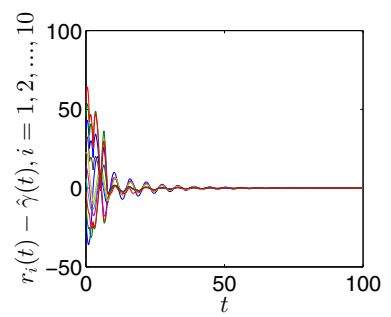

(a)

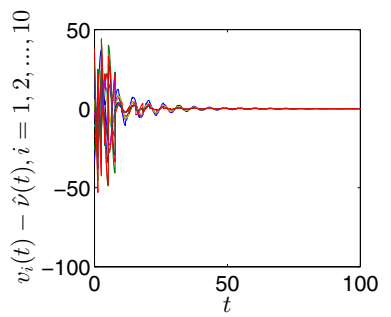

(b)
Fig. 6. The time evolution of tracking errors $r_{i}-\hat{\gamma}$ and $v_{i}-\hat{\nu}$ with a fixed commnuication topology $\mathcal{G}$ via tracking a moving object (10) with initial value $\hat{\gamma}(0)=10$ and $\hat{\nu}(0)=-10$, where $\alpha=1.2, T_{k}=1.3, \sigma^{2}=0.003$ and $\mu=0.2$.

\section{Conclusion}

In this paper, an effective algorithm has been proposed for synchronizing a set of coupled harmonic oscillators by using some sampled data that contains measurement noise. We have showed that the oscillators can be synchronized even when the velocity information is exchanged only at discrete-time instants and discussed the convergence of the algorithm for directed networks with either fixed or switching topologies in the presence or absence of leaders. We have also established the sufficient conditions for which the coupled harmonic oscillators could attain synchronized oscillatory motions containing noise. Some numerical examples are given to illustrate the effectiveness of the theoretical results.

\section{References}

Ballard, L., Cao, Y., and Ren, W. (2010). Distributed discretetime coupled harmonic oscillators with application to synchronised motion coordination. IET Control Theory Applications, 4(5):806-816.

Boccaletti, S., Kurths, J., Osipov, G., Valladares, D. L., and Zhou, C. S. (2002). The synchronization of chaotic systems. Physics Reports, 366(1-2):1-101.

Casau, P., Sanfelice, R. G., Cunha, R., Cabecinhas, D., and Silvestre, C. (2015). Robust global trajectory tracking for a class of underactuated vehicles. Automatica, 58:90-98.

Chiorescu, I., Bertet, P., Semba, K., Nakamura, Y., Harmans, C., and Mooij, J. (2004). Coherent dynamics of a flux qubit coupled to a harmonic oscillator. Nature, 431(7005):159-162.
Dörfler, F. and Bullo, F. (2014). Synchronization in complex networks of phase oscillators: A survey. Automatica, 50(6):15391564 .

Li, Z. and Ding, Z. (2015). Distributed adaptive consensus and output tracking of unknown linear systems on directed graphs. Automatica, 55:12-18.

Olfati-Saber, R., Fax, J. A., and Murray, R. M. (2007). Consensus and cooperation in networked multi-agent systems. Proceedings of the IEEE, 95(1):215-233.

Petersen, K. B. and Pedersen, M. S. (2012). The Matrix Cookbook. Technical University of Denmark.

Ren, W. (2008). Synchronization of coupled harmonic oscillators with local interaction. Automatica, 44(12):3195-3200.

Ren, W. and Beard, R. (2005). Consensus seeking in multiagent systems under dynamically changing interaction topologies. IEEE Transactions on Automatic Control, 50(5):655-661.

Shen, B., Wang, Z., and Liu, X. (2012). Sampled-data synchronization control of dynamical networks with stochastic sampling. IEEE Transactions on Automatic Control, 57(10):26442650 .

Sun, W., Lü, J., Chen, S., and Yu, X. (2014). Synchronisation of directed coupled harmonic oscillators with sampled-data. IET Control Theory \& Applications, 8:937-947.

Sun, W., Yu, X., Lü, J., and Chen, S. (2015). Synchronization of coupled harmonic oscillators with random noises. Nonlinear Dynamics, 79(1):473-484.

Tanner, H. G., Jadbabaie, A., and Pappas, G. J. (2007). Flocking in fixed and switching networks. IEEE Transactions on Automatic Control, 52(5):863-868.

Wang, J., Xu, C., Feng, J., Chen, M., Wang, X., and Zhao, Y. (2015a). Synchronization in moving pulse-coupled oscillator networks. IEEE Transactions on Circuits and Systems I, 62(10):2544-2554.

Wang, L., Chen, M. Z. Q., and Wang, Q.-G. (2015b). Bounded synchronization of a heterogeneous complex switched network. Automatica, 56:19 - 24 .

Wang, X. and Chen, G. (2002). Synchronization in scale-free dynamical networks: robustness and fragility. IEEE Transactions on Circuits and Systems I, 49(1):54-62.

Wen, G., Yu, W., Chen, M. Z. Q., Yu, X., and Chen, G. (2014). $H_{\infty}$ pinning synchronization of directed networks with aperiodic sampled-data communications. IEEE Transactions on Circuits and Systems I, 61(11):3245-3255.

Yang, T. (2001). Impulsive control theory, volume 272 of Lecture Notes in Control and Information Sciences. Springer.

Zhang, H. and Shi, Y. (2012). Observer-based $\mathcal{H}-\infty$ feedback control for arbitrarily time-varying discrete-time systems with intermittent measurements and input constraints. Journal of Dynamic Systems, Measurement, and Control, 134(6):061008.

Zhang, H. and Zhou, J. (2012). Synchronization of sampleddata coupled harmonic oscillators with control inputs missing. Systems \& Control Letters, 61(12):1277-1285.

Zhang, Y., Yang, Y., and Zhao, Y. (2013). Finite-time consensus tracking for harmonic oscillators using both state feedback control and output feedback control. International Journal of Robust and Nonlinear Control, 23(8):878-893.

Zhang, Z., Xu, S., and Zhang, B. (2015). Exact tracking control of nonlinear systems with time delays and dead-zone input. Automatica, 52:272-276.

Zhou, J., Zhang, H., Xiang, L., and Wu, Q. (2012). Synchronization of coupled harmonic oscillators with local instantaneous interaction. Automatica, 48(8):1715-1721.

Zhou, J., Zhang, H., Xiang, L., and Wu, Q. (2013). Sampled-data 
synchronization of coupled harmonic oscillators with controller failure and communication delays. Theoretical 83 Applied Mechanics Letters, 3(6):62997. 